EPJ Web of Conferences 116, 05005 (2016)

DOI: $10.1051 /$ epjconf/201611605005

(C) Owned by the authors, published by EDP Sciences, 2016

\title{
The Trigger and Data Acquisition System for the KM3NeT neutrino telescope
}

\author{
Carmelo Pellegrino ${ }^{1,2, \mathrm{a}}$ and Tommaso Chiarusi ${ }^{1, \mathrm{~b}}$ on behalf of the KM3NeT Collaboration \\ ${ }^{1}$ INFN Sezione di Bologna,V.le Berti Pichat 6/2, 40127 Bologna, Italy \\ ${ }^{2}$ Dipartimento di Fisica e Astronomia Università di Bologna, V.le Berti Pichat 6/2, 40127 Bologna, \\ Italy
}

\begin{abstract}
KM3NeT is a large research infrastructure in the Mediterranean Sea that includes a network of deep-sea neutrino telescopes. The telescopes consist of vertical detection units carrying optical modules, whose separation is optimised according to the different ranges of neutrino energy that shall be explored. Two building blocks, each one made of 115 detection units, will be deployed at the KM3NeT-IT site, about $80 \mathrm{~km}$ from Capo Passero, Italy, to search for high-energy neutrino sources (ARCA); another building block will be installed at the KM3NeT-Fr site, about $40 \mathrm{~km}$ from Toulon, France, to study the hierarchy of neutrino masses (ORCA). The modular design of the KM3NeT allows for a progressive implementation and data taking even with an incomplete detector. The same scalable design is used for the Trigger and Data Acquisition Systems (TriDAS). In order to reduce the complexity of the hardware inside the optical modules, the "all data to shore" concept is adopted. This implies that the throughput is dominated by the optical background due to the decay of ${ }^{40} \mathbf{K}$ dissolved in the sea water and to the bursts of bioluminescence, about 3 orders of magnitude larger than the physics signal, ranging from $20 \mathrm{Gbps}$ to several hundreds Gbps, according to the number of detection units. In addition, information from the acoustic positioning system of the detection units must be transmitted. As a consequence of the detector construction, the on-shore DAQ infrastructure must be expanded to handle an increasing data-rate and implement an efficient fast data filtering for both the optical and acoustic channels. In this contribution, the Trigger and Data Acquisition System designed for the Phase 1 of KM3NeT and its future expansion are presented. The network infrastructure, the shore computing resources and the developed applications for handling, filtering and monitoring the optical and acoustic data-streams are described.
\end{abstract}

\section{Introduction}

KM3NeT [1] deep-sea research infrastructure consisting of a network of neutrino telescopes will be installed in two sites in the Mediterranean Sea: off Toulon at a depth of about $2500 \mathrm{~m}$, France, the ORCA (Oscillation Research with Cosmics in the Abyss) detector will study the neutrino mass hierarchy, using 115 string-like [2] Detection Units (DUs); off Portopalo di Capo Passero at a depth of 3500 m, Sicily,

\footnotetext{
a e-mail: carmelo.pellegrino@bo.infn.it

b e-mail: tommaso.chiarusi@bo.infn.it
}

This is an Open Access article distributed under the terms of the Creative Commons Attribution License 4.0, which permits unrestricted use, distribution, and reproduction in any medium, provided the original work is properly cited. 


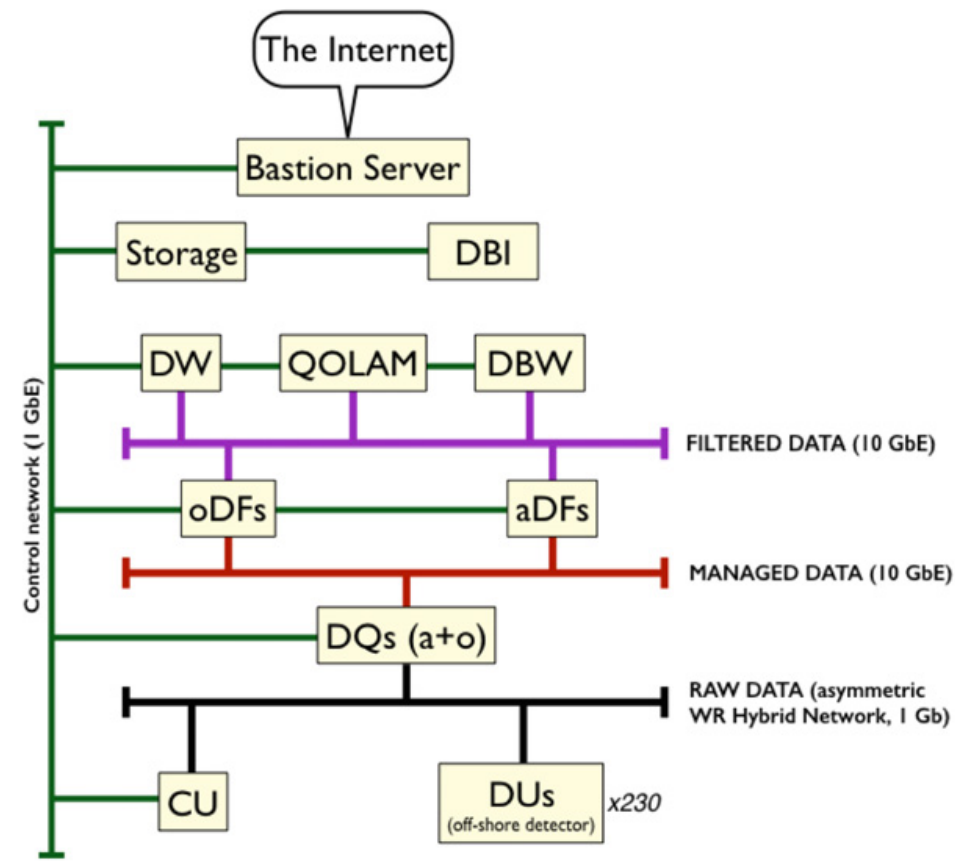

Figure 1. Sketch of the network topology of the KM3NeT detector and shore-station infrastructure. For a detailed description see the text.

the ARCA (Astronomy Research with Cosmics in the Abyss) detector will study ultra high energy neutrinos expected by astrophysical and cosmologic cosmic rays sources. ARCA will be made by about 230 string-like and 8 tower-like [3, 4] DUs arranged in a $\sim 100$ meters-spaced grid. Each string carries 18 Digital Optical Modules (DOMs) [5, 6], which are vertically separated by 6 and 36 meters in ORCA and ARCA, respectively. Each DOM is a $17^{\prime \prime}$ diameter glass sphere containing $313^{\prime \prime}$ photomultiplier tubes. The detection principle is based on the collection of the Cherenkov light emitted along the path of charged particles produced by neutrino interactions in the detector vicinity.

Due to the high optical background caused by $\beta$ decay of ${ }^{40} \mathrm{~K}$ and bioluminescence phenomena in sea water, an on-line trigger is needed. In order to minimise the complexity of the underwater electronics, no hardware trigger is present and the all-data-to-shore approach is used: all detected optical signals are sent to shore via a computer network which comprehends both the shore-station and the detector. In the final configuration of the experiment, the expected data throughput from both sites is up to $500 \mathrm{Gbps}$.

\section{Network topology}

The DOMs, i.e. the off-shore detector, and the shore-station computing infrastructure are part of a computing network subdivided in 3 main subnetworks (see Fig. 1):

- Raw data network: aims at connecting the off-shore detector elements (DOMs and DU bases) with both the computers dedicated to the aggregation and distribution of data (DataQueue, see the next section) and the ControlUnit of the experiment;

- Managed data network: aims at connecting the DataQueues with the data filtering infrastructure;

- Filtered data network: is devoted to the transportation of post trigger data. 
The Raw data network represents the main networking peculiarity of the experiment. It makes use of a White Rabbit [8] asymmetric network, which also provides for sub-nanosecond time synchronisation. The asymmetry comes from the physical topology of the optical links between the underwater detector elements and the on-shore station. Every single DOM has a unidirectional $1 \mathrm{~Gb} / \mathrm{s}$ uplink to reach the on-shore station delivering the acquired data and the slow control (SC) replies. On the other side, the on-shore station has a unique unidirectional $1 \mathrm{~Gb} / \mathrm{s}$ downlink to steer all the DOMs. This is called the broadcast and is shared by every single DOM, so that when a DOM receives a packet, all other DOMs also receive it. This topology highly reduces the communication resources cost (i.e. the number of optical fibres), but it requires a specific customisation of the communication elements as the switches and the DOMs.

The Managed and Filtered data networks are standard Ethernet networks that provide a data transfer speed of $10 \mathrm{Gbps}$.

A $1 \mathrm{GbE}$ Control network allows the connection of all the shore-station computing elements with the ControlUnit and provides access to the Internet through a Bastion Server, which protects the internal networks.

\section{The KM3NeT TriDAS}

The KM3NeT Trigger and Data Acquisition System [7] (TriDAS) is the software and computing infrastructure deputed to the read-out, aggregation and filtering of all the detector data. All TriDAS components are connected through the network described in Sect. 2.

Each DOM carries $313^{\prime \prime}$ PMTs and a piezo-electric acoustic sensor that produce the two main data streams from off-shore: the optical and acoustic data streams, respectively. The DOM digitises the signals from the PMTs as photon hits - defined by the integrated electric charge of the PMT signal over a certain threshold, the crossing time and the duration of the signal over the threshold - and collects them into optical data frames. Acoustic signals reaching the piezo-electric sensor are sampled at a constant frequency and with a proper resolution - both tunable from on-shore - and compose an acoustic data frame. Once a data frame is completed, the DOM sends it to shore using a UDP/IP protocol.

On-shore, the first data processing stage is represented by the DataQueue (DQ) farm which is in charge of the aggregation of unfiltered data and of their distribution to the computers dedicated to the on-line analysis and trigger, namely DataFilters (DFs). For each data stream a specific implementation of the DF exists, called AcousticDataFilter (aDF) and OpticalDataFilter (oDF) for acoustic and optical data streams, respectively. Each DQ is responsible for the collection of the data coming from a sector (i.e. a subset of DOMs) of the detector. Each oDF receives from all the DQs a bunch of data belonging to a precise time interval called Time Slice (TS). The duration of a TS is fixed, typically $100 \mathrm{~ms}$. In this way, each oDF has a complete snapshot, limited to the TS duration, of the status of the whole detector. Triggered data coming out from the oDFs are sent to the DataWriter (DW) that collects them on permanent storage as ROOT [9] files. Each aDF, differently from the optical case, receives all data from a single DQ continuously, thus having the complete data time series from a subset of the acoustic sensors. The aim of the aDF is to provide on-line reconstruction of the Time Of Arrival (TOA) of the acoustic signals emitted by the acoustic beacons of the acoustic positioning system [10]. The output of all the aDFs is collected by the DataBaseWriter (DBW, see below) and written to a database.

The ControlUnit (CU) [11], which represents the user interface to the detector, aims at coordinating the TriDAS and operating the DOMs through a dedicated SlowControl (SC) protocol. It is composed of the following sub-systems:

- Master Control Program (MCP): is the main user interface and allows to schedule data taking of the experiment;

- TriDAS Manager (TM): steers the on-shore computing facility, setting up the TriDAS processes; 
- Detector Manager (DM): controls and configures the off-shore detector;

- DataBaseInterface (DBI): provides local database caching and synchronisation with the remote $\mathrm{DB}$;

- DataBaseWriter (DBW): collects slow-control data and TOAs from the aDFs and writes them to the DB.

The Quasi On-Line Analysis and Monitoring (QOLAM) [12] is a suite of programs that perform fast analysis on a subset of the collected post-trigger data, visualisation and monitoring. It is composed by:

- ROyWeb wich is a web-based program that allows to plot monitored parameters as time series, histograms or as synoptic plots;

- Rainbow Alga which is the 3D event display;

- ROyFit is the fast muon reconstruction software, based on an ANTARES algorithm [13].

\section{Conclusions}

The KM3NeT experiment will perform continuous data taking for at least 20 years in two sites, reaching a total throughput up to $500 \mathrm{Gbps}$ per site. The construction and operation of the detector involves several challenging techniques, such as multi-PMT optical modules, asymmetric network and WhiteRabbit standard. The TriDAS is a custom designed system for data acquisition and triggering and has been designed to be modular and to scale with a growing up detector.

On December 3rd 2015 the first string of the ARCA detector has been deployed in the abyssal site of Capo Passero, in Sicily. Since then, the on-line Trigger and Data Acquisition System has been continuously running at the Portopalo control station, collecting data according to expected performances.

\section{References}

[1] KM3NeT Collaboration website, http://www .km3net.de

[2] Biagi S. for the KM3NeT Collaboration, Operation and results of the prototype KM3NeT Detection Unit, proceedings of the 5th Roma International Conference on Astroparticle Physics, Noto, Italy

[3] Aiello S. et al. (NEMO Collaboration), Measurement of the atmospheric muon depth intensity relation with the NEMO Phase-2 tower, Astropart. Phys. 66, 2015, 1-7

[4] M. Favaro et al., these proceedings

[5] Circella M., The Digital Optical Module (DOM) for the KM3NeT Detector, proceedings of the 33nd ICRC, Rio de Janeiro, 2013

[6] D. Real et al., these proceedings

[7] Chiarusi T. et al. (NEMO Collaboration) KM3NeT: R\&D and technical solutions for the next generation underwater neutrino telescope, proceedings of ICHEP 2014

[8] WhiteRabbit website, http://www.ohwr.org/projects/white-rabbit

[9] CERN http://www.root.cern.ch

[10] F. Simeone et al., these proceedings

[11] C. Bozza et al., these proceedings

[12] T. Gal et al., these proceedings

[13] J.A. Aguilar et al. (ANTARES Collaboration), A fast algorithm for muon track reconstruction and its application to the ANTARES neutrino telescope, Astropart. Phys. 34, 2011, 652-662 